\title{
El impacto de las prácticas pre profesionales en la cultura tributaria de los estudiantes de la Universidad Católica de Cuenca
}

\section{The impact of pre professional practices in the tax culture of students of the Catholic University of Cuenca}

\author{
URL: http://revistas.uta.edu.ec/erevista/index.php/bcoyu/article/view/885 \\ DOI: http://dx.doi.org/10.31164/bcoyu.24.2020.885 \\ Patricio Mendieta- Andrade ${ }^{1}$; Janice Ordoñez- Parra ${ }^{2}$; Gina Cuadrado- Sánchez ${ }^{3}$ \\ Fecha de recepción: 28 de noviembre de 2019 \\ Fecha de aceptación: 14 de enero de 2020
}

\begin{abstract}
Resumen
El presente trabajo analiza el impacto que ha tenido el Proyecto Núcleo de Apoyo Contable y Fiscal de la Universidad Católica de Cuenca en la cultura tributaria de los estudiantes participantes en el mismo, en el período 2016-2018; para lo cual, se utilizó la información de la base de datos del Proyecto y el resultado de la encuesta plantada a los estudiantes; siendo que, se evidenció un impacto positivo en los conocimientos, percepciones y actitudes frente a los impuestos por parte de los estudiantes luego de concluida su participación en el Proyecto, identificándose el apoyo de los docentes, como uno de los factores que percibieron los estudiantes, como el más influyente en el impacto identificado.
\end{abstract}

Palabras clave: Cultura tributaria, vinculación con la comunidad, evaluación de impacto.

\section{Abstract}

This paper analyzes the impact that the Accounting and Fiscal Support Core Project of the Catholic University of Cuenca has had on the tax culture of the students participating in it in the 2016-2018 period; for which the information from the Project database and the results of the survey planted to the students were used; being that, a positive impact on the knowledge, perceptions and attitudes towards taxes on the part of the students was evidenced after their participation in the Project was concluded, identifying the support of the teachers, as one of the factors that the students perceived, as the most influential in the identified impact.

Keywords: Tax culture, community engagement, impact assessment.

\footnotetext{
${ }^{1}$ Universidad Católica de Cuenca. Unidad Académica de Administración.Cuenca-Ecuador. Email: pmendietaa@ucacue.edu.ec. ORCID: https://orcid.org/0000-0001-95964344

${ }^{2}$ Universidad Católica de Cuenca. Unidad Académica de Administración. Cuenca-Ecuador. Email: jordonezp@ucacue.edu.ec. ORCID: https://orcid.org/0000-0002-50022203

${ }^{3}$ Universidad Católica de Cuenca. Unidad Académica de Administración. Cuenca-Ecuador. Email: gcuadrado@ucacue.edu.ec. ORCID: https://orcid.org/0000-0002-31879586
} 


\section{Introducción}

Según cifras de la Proforma Presupuestaria 2019, presentada por el Ministerio de Economía y Finanzas (2018), los impuestos representan aproximadamente el $44 \%$ de los ingresos totales con los que el estado pretende financiar el total de su gasto en el presente año; por lo que, se evidencia la dependencia que tiene la economía nacional de este rubro, siendo imperativo que la academia aporte con profesionales idóneos, con formación sólida tanto en conocimientos como con fundamentos éticos capaces de contribuir al desarrollo de la sociedad desde el punto de vista de la fiscalidad.

Por lo anotado, la Universidad Católica de Cuenca (UCACUE) en el año 2016 firmó un Acuerdo Interinstitucional con el Servicio de Rentas Internas (SRI), por el cual se comprometieron las partes a instituir un Núcleo de Apoyo Contable Fiscal (NAF) en el Alma Mater, como un espacio en el que los estudiantes de la Unidad Académica de Administración de Empresas puedan desarrollar sus Prácticas Pre Profesionales de Vinculación con la Sociedad (PPPVS), enfocándose en brindar asesoramiento gratuito a las personas naturales y microempresarios de la ciudad de Cuenca, en el cumplimiento de sus obligaciones fiscales y el acceso a sus beneficios tributarios; siendo de esta manera, actores propositivos en el fortalecimiento de la cultura tributaria de los ciudadanos del cantón.

En línea con lo anotado, el presente artículo tiene como objetivo poner en evidencia el impacto que tuvieron las PPPVS realizadas por los estudiantes en el NAF de la UCACUE en el período 2016 a 2018, partiendo de la hipótesis de que las actividades que desarrollaron los estudiantes y el contacto directo con la realidad de los contribuyentes fortaleció su cultura tributaria, siendo esta la variable dependiente del trabajo y definida, en sí, como el conjunto de actitudes, conocimientos y percepciones frente a los impuestos (Aya \& Báez, 2017); por consiguiente, y para la consecución del propósito planteado se analizó la información de la base de datos del Proyecto y otros datos provenientes de las encuestas aplicadas a los estudiantes.

Se destaca que la relevancia del estudio presentado radica, en primer lugar, que el mismo constituye una evaluación al Proyecto NAF, a fin de reconocer sus aciertos e identificar aspectos deficientes que se pudieran mejorar en el propósito de conseguir los objetivos planteados en el mismo y, por otro lado, visibilizar al Proyecto como uno de los tantos aportes se hace desde la UCACUE para la construcción de una sociedad justa y solidaria, dando cumpliendo a su apostolado de "Comunidad Educativa al Servicio del Pueblo".

Finalmente cabe anotar que, posterior a esta nota introductoria se presentan los aspectos más relevantes obtenidos en la revisión bibliográfica pertinente a la investigación planteada, para luego especificar la metodología empleada en la misma, con la cual se obtuvieron los resultados, que se presentan en apartado tres; siendo éstos la base para la especificación de las correspondientes conclusiones del caso.

\section{Marco teórico}

En el marco de la cooperación entre la Unión Europea y América Latina, el programa EUROsociAL se constituye como eje en la promoción de la cohesión social, fortaleciendo la institucionalidad de los países, a través del diseño, reforma e implementación de políticas gubernamentales enfocadas en el bienestar, igualdad y solidaridad de los conformantes de las sociedades en nuestra región (EUROsociAL, 2017). Por lo expuesto en el año 2014 se reúnen en Lima, Perú, los representantes de diferentes universidades y dependencias gubernamentales relacionadas a la administración tributaria de la Región, incluidas las de nuestro país, donde acuerdan formalmente constituir la Red NAF como un espacio de integración, enfocado en compartir experiencias y promotor de la creación de los NAF en cada país (Red NAF, 2014).

Según datos de EUROsociAL (2018, pág. 22) en el año 2017 se registraron, en la referida Red, 476 NAF con convenio vigente con sus respectivos órganos de administración tributaria en 9 países; siendo que el $63,0 \%$ de éstos se encuentran en Brasil, el 11,1\% en Colombia y el $9,5 \%$ en México. Cabe anotar que, los primeros NAF en América Latina se constituyeron en Brasil dada la iniciativa del órgano nacional administrador de los impuestos, la Receita Federal, siendo que, según datos oficiales, en éste país en la actualidad existen formalmente más de $300 \mathrm{NAF}$ en funcionamiento bajo convenio con diferentes universidades, ofreciendo servicios contables gratuitos, tanto para personas naturales y jurídicas de menor tamaño (Receita Federal, s.f).

Ya en el plano nacional, desde el año 2015 el SRI viene fomentando la implementación de los NAF en nuestro país como centros de información gratuitos sobre temas tributarios y contables para personas naturales no obligadas a llevar contabilidad y pequeñas empresas; los cuales se enmarcan en convenios interinstitucionales entre esta Institución y las diferentes universidades del país (SRI, 2018). Es así que, actualmente existen 25 NAF en funcionamiento en todo el Ecuador, en ciudades como Esmeraldas Tulcán, Ibarra, Ambato, Latacunga Riobamba, Santo Domingo, Jipijapa, La Libertad, Cuenca, Azogues, Loja, Machala, Guayaquil y Quito; siendo que, en la ciudad de Cuenca se han implementado tres unidades NAF, auspiciados por la Universidad Politécnica Salesiana, Universidad del Azuay y por la Universidad Católica de Cuenca (SRI, 2018).

Tomando como referencia los datos presentados por el SRI (2018) en su página web, los principales servicios que brindan los NAF son:

- Información general contable y para la presentación oportuna de obligaciones y trámites tributarios (RUC, facturación, declaraciones y anexos, vehículos, etc.)

- Información general sobre obligaciones tributarias pendientes. 
- Información general sobre el Régimen Impositivo Simplificado (RISE).

- Información general sobre medios de pago de impuestos y deudas tributarias.

- Información general sobre devolución de impuestos a Adultos Mayores y Discapacitados.

- Información general para la utilización de los servicios en línea del SRI.

De la misma manera, en cuanto a los servicios contables que prestan los NAF se reconoce la capacidad que tienen los estudiantes de poner en práctica, en estos centros, sus conocimientos en temas contables y otros inherentes a la administración de empresas, tales como, según la experiencia de la Universidad de la Costa (s.f) se distinguen el asesoramiento en la elaboración de contratos de trabajo, roles de pagos a empleados, liquidación de beneficios sociales, asesoría en sistemas contables de costos y preparación de estados financieros.

Cabe puntualizar que los NAF no tienen las funciones como centros de atención de la Administración Tributaria y no se constituyen como sustitutos de las oficinas de contabilidad (Red NAF, 2015)

Un punto importante, que según destaca el Servicio de Administración Tributaria de México (s.f) y coincide con la realidad ecuatoriana, es que los estudiantes y docentes que brindan los servicios en el NAF son previamente capacitados, bien sea en las aulas universitarias durante sus estudios profesionales, así como por los entidades administradoras de los tributos, a fin de cumplir de forma cabal con el objetivo de apoyar en el cumplimiento de las obligaciones tributarias.

Finalmente, en concordancia con lo anotado por la Pontificia Universidad Javeriana de Bogotá (s.f), los NAF surgen de un esfuerzo conjunto entre la Universidad y la Administración Tributaria (la Dirección de Impuestos y Aduanas en el caso colombiano), con el fin de fortalecer la cultura tributaria de los individuos conformantes de una sociedad, siendo el objetivo que éstos comprendan y se identifiquen como ciudadanos con derecho y deberes. Es así, que se entiende como cultura tributaria "un conjunto de valores, conocimientos y actitudes compartidos por los miembros de una sociedad respecto a la tributación y la observancia de las leyes que la rigen" (Mendoza, Cano, Robles, \& Ramiréz, 2016).

\section{EI NAF de la Universidad Católica de Cuenca}

Como punto de partida de este apartado se anota que la Ley Orgánica de Educación Superior en su Artículo 8 define como uno de los fines de la educación superior el "contribuir en el desarrollo local y nacional de manera permanente, a través del trabajo comunitario o vinculación con la sociedad"; siendo que en el Artículo 125 de la mencionada Ley se especifica que "las instituciones del Sistema de Educación Superior realizarán programas y cursos de vinculación con la sociedad guiados por el personal académico" (Asamblea Nacional, 2018); por lo cual, se reconoce el marco legal que sustenta la vinculación con la comunidad como uno de los ejes de ejes fundamentales de la Instituciones de Educación Superior y una de las funciones sustantivas de las mismas, junto a la docencia y la investigación.

El Reglamento de Prácticas Pre Profesionales de la Universidad Católica de Cuenca, establece que estas se constituyen con el fin de integrar la teoría con la práctica, en el proceso de aprendizaje, con actividades que permitan la aplicación del conocimiento y el desarrollo de los conocimientos, habilidades y destrezas de los estudiantes; siendo más concretos, las PPPVS se enfocan en el servicio de la sociedad, y su planificación se ejecuta durante los cuatro últimos ciclos de la carrera con un mínimo de cientos sesenta horas (Secretaria General Universidad Católica de Cuenca, 2015). Es así, que se destacan, entre otros, como objetivo de las PPPVS:

Articular a los proyectos de vinculación con la sociedad los ejes estratégicos de desarrollo contenidos en el Plan Nacional del Buen Vivir, las necesidades y demandas de los territorios, las tendencias del conocimiento y la profesión, con miras a garantizar su calidad y pertinencia (Secretaría General - Universidad Católica de Cuenca, 2017).

En el contexto anotado, la Universidad Católica de Cuenca, establece el Acuerdo de Cooperación Interinstitucional entre la Universidad Católica de Cuenca y el Servicio de Rentas Internas, por el cual se propicia la creación del NAF de la UCACUE, como un centro gratuito de difusión del conocimiento contable y tributario, instruyendo a los beneficiarios sobre el sentido social de los impuestos y el cumplimiento de sus deberes formales como contribuyentes (UCACUE, 2016).

Dados los antecedentes presentados, el Proyecto de Vinculación con la Colectividad Núcleo de Apoyo Contable de las Carreras de Ingeniería Comercial e Ingeniería en Contabilidad de la Unidad Académica de Administración de la Universidad Católica de Cuenca, marca como fecha de inicio de sus actividades el nueve de mayo de 2016 , estableciéndose una duración del mismo de cuatro años (UCACUE, 2016). Se anota que el diseño del Proyecto NAF se sustenta en el Objetivo 8 del Plan Nacional del Buen vivir 2013-2017, es decir "consolidar el sistema económico social y solidario", concretamente con la Política 8.1: "fortalecer la progresividad y la eficiencia del sistema tributario" (Secretaria Nacional de Planificación y Desarrollo, 2013).

Cabe anotar, que según los datos que constan en el documento del Proyecto de Vinculación con la Comunidad Núcleo de Apoyo Contable y Fiscal (2016), el objetivo de desarrollo del Proyecto es "contribuir al fortalecimiento de la cultura tributaria en el cantón Cuenca"; siendo que de forma concreta, en la citada fuente y dentro de sus tres objetivos específicos se destaca que el NAF se constituirá a fin de: 
Generar un espacio en el cual los estudiantes de la Unidad Académica de Administración de la UCACUE puedan poner en práctica sus conocimientos inherentes a la contabilidad y la tributación, con lo cual cumplan con su requisito de prácticas pre profesionales y vinculación a la sociedad.

Finalmente, se reconoce, en el citado documento, como uno de los resultados esperados del Proyecto que 138 estudiantes participen en el mismo durante sus cuatro años de duración, esperando una calificación promedio de 80 sobre 100 en cuanto a su satisfacción del proceso de PPPVS experimentadas en el Proyecto NAF de la UCACUE.

\section{Metodología}

El presente trabajo se planteó, por un lado, de carácter descriptivo no experimental del tipo transversal, pues exhibe la realidad de los estudiantes una vez concluido su período de prácticas en el NAF; por otro lado, al tratar de identificar el impacto del NAF en la cultura tributaria de los estudiantes, se identifican en el estudio rasgos de una investigación del tipo analítico correlacional, pues se busca evidenciar la asociación o correlación entre diferentes variables partiendo de una hipótesis pre establecidas (Rojas, 2015)

De la misma manera, se anota que la presente investigación se empleó, el enfoque cualitativo, donde el trabajo se enmarcó en un diseño etnográfico con el que, según Salgado (2007, pág. 72), se busca "describir y analizar ideas creencias, significados, conocimientos y prácticas de grupos, culturas y comunidades"; pues, con éste estudio se identificó los rasgos que distinguen la cultura tributaria de los estudiantes que participaron en el Proyecto NAF, para lo cual se aplicó una encuesta, en la cual mediante el empleo de la Escala de Likert se cuantificó las percepciones del grupo estudiado. Cabe anotar que, para el análisis planteado, también se utilizaron los datos de las bases propias del Proyecto.

Por otro lado, el estudio al pretender evidenciar el efecto que tuvieron las PPPVS en la cultura tributaria de los estudiantes que participaron en el NAF, se enmarcó bajo los parámetros de una investigación correlacional, con el objetivo de "conocer la relación o grado de asociación que existe entre dos o más conceptos, categorías o variables en un contexto en particular" (Sampieri, Fernández, \& Baptista, 2010, pág. 81). Es así que, en primera instancia se determinó que los datos obtenidos se definen bajo una escala ordinal, los cuales surgen a partir de una operación de ordenamiento que representan categoría con un orden asociado (Merli, 2010), siendo éstos presentados en los correspondientes gráficos y tablas de frecuencias, para posteriormente proceder a evaluar el grado de asociación de los resultados obtenidos en la encuesta mediante la utilización del coeficiente de Correlación de Spearman, el cual constituye una "medida de asociación lineal que utiliza los rangos o números de orden, de cada grupo de sujetos y compara dichos rangos" (Martínez, Martínes, Martínez, Pérez, \& Cánovas, 2009).

Para el trabajo investigativo se definió como población o universo a los 78 estudiantes que participaron en el NAF de la UCACUE desde el inicio de sus funciones en mayo de 2016 hasta el mes de febrero de 2018. De igual forma, se anota que en la investigación se aplicó la encuesta a una muestra definida de 36 estudiantes, la cual refiere un $90 \%$ de confianza, una proporción del $50 \%$ y un margen de error del 10\%; para tal efecto, la conformación de la muestra se lo hizo de forma aleatoria entre los conformantes de la población ya definida.

\section{Resultados}

En primera instancia se anota que el Proyecto de Vinculación con la Sociedad Núcleo de Apoyo Contable y Fiscal (NAF) de la UCACUE inicia sus actividades en el mes de mayo del 2016 proyectándose una duración de cuatro años. Para el presente estudio se consideró como población a los 78 estudiantes que participaron en el Proyecto desde sus inicios hasta el mes de febrero de 2019, los cuales pertenecen a las diferentes carreras de la Unidad Académica de Administración, en las proporciones que se presentan en la Figura 1:

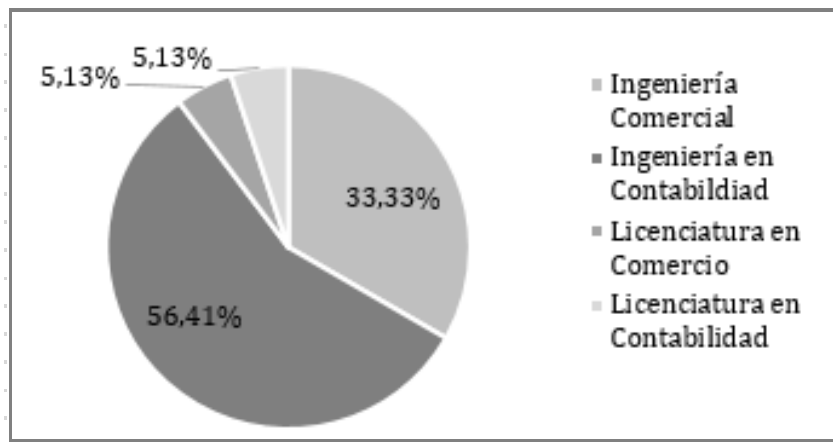

Fuente: Elaboración propia en base a la base de datos del Proyecto NAF de la UCACUE Figura 1. Porcentaje de alumnos participantes en el Proyecto NAF por carreras.

Según se aprecia en la Figura 1, el mayor porcentaje de estudiantes que participaron en el NAF de la UCACUE durante el período de estudio pertenecieron a la Carrera de Ingeniería en Contabilidad, seguido de los estudiantes de la Carrera de Ingeniera Comercial; se reconoce que una baja participación de los alumnos de las Licenciaturas, este hecho se explica dado a que estas son carrera sometidas a rediseños y por tanto son los primeros estudiantes de los sextos ciclos los que se insertaron en el Proyecto.

Al analizar la proporción del genero de los estudiantes que participaron en el NAF se reconoce que el $73,08 \%$ fueron de género femenino y el $26,92 \%$ masculino, con lo cual se verifica los resultados del trabajo de Mantilla, Galarza y Zamora (2017), en el que se pone en evidencia la superioridad en la proporción del alumnado femenino en las carreras de índole social-humanístico. 
Al verificar el nivel de mejora de los conocimientos de los aspectos prácticos inherentes al cumplimiento de los tributos por parte de los estudiantes luego de sus prácticas, se puso en evidencia que en promedio los estudiantes califican en promedio, en la escala ya anotada del 1 al 10, con 8,80 la mejoría en éste aspecto, siendo la desviación típica de 1,53; es así que, se reconoce que un $44,44 \%$ califica como muy alta la mejora en el aspecto específico planteado, según se muestra en la Figura 4

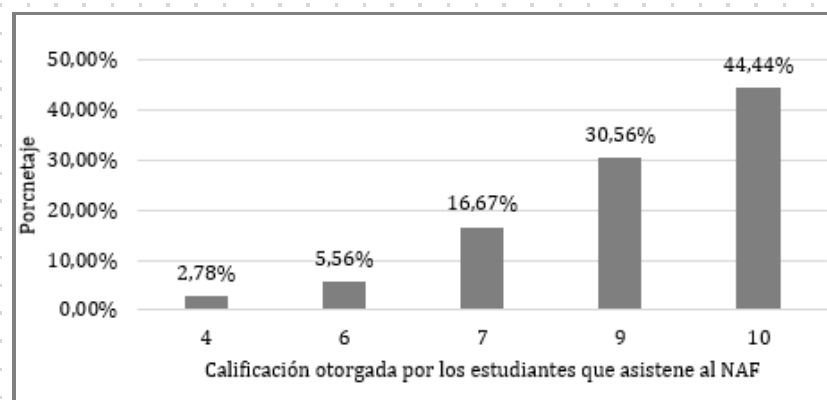

Fuente: Elaboración propia en base a los resultados de la encuesta de investigación

Figura 4. Calificación otorgada por los estudiantes que participan en el NAF de la UCACUE referente a la mejora en sus conocimientos sobre los procedimientos inherentes al cumplimiento de las obligaciones tributarias luego de sus prácticas

Continuando con el análisis, se propuso estimar el grado de correlación existente entre la percepción que refieren los estudiantes sobre la mejora de sus conocimientos teóricos y aquellos conocimientos prácticos adquiridos durante sus prácticas en el NAF; para lo cual, se aplicó el coeficiente de correlación de Spearman, donde el estadístico asumió un valor rho=0,940 ( $p=0,000)$; por lo que se reconoce la significancia estadística de la correlación de las dos variables planteadas; siendo que los valores evidencian, en base a los apuntes de Martínez et al. (2009), una correlación fuerte y positiva, es decir que mientras mayor es la percepción de la mejora de los conocimientos prácticos mayor será la percepción de la mejora de los conocimientos teóricos. Por lo expuesto se pone en evidencia que las actividades prácticas desarrollados por los estudiantes en el NAF tienen un efecto positivo en la mejora de sus conocimientos teóricos sobre aspectos tributarios y contables; con los que se reconoce el impacto positivo del proyecto en el proceso de aprendizaje de los estudiantes.

De la misma manera, a fin de evaluar el impacto que tuvieron las PPPVS desarrolladas por los estudiantes en el NAF de la UCACUE en su cultura tributaria, se consultó mediante el cuestionario planteado, su opinión referente a aspectos relacionados a la fiscalidad en el país, lo cual nos posibilitará definir cuál es su percepción y actitud frente a los impuestos luego de haber concluido su participación en el Proyecto.

Es así que, el $66,67 \%$ de los encuestados afirma que luego de concluidas sus prácticas en el NAF de la UCACUE, está de acuerdo con la afirmación de que el cumplimiento de las obligaciones tributarias es un deber de todos los miembros de una sociedad, siendo que un $5,56 \%$ está en descuerdo y un $5,56 \%$ se mantiene neutro frente a la afirmación planteada. Por lo expuesto al tener el mayor porcentaje de respuestas concordantes con el enunciado especificado, se cumple con uno de los objetivos de la educación, que según Quintanilla (2012) sería romper el círculo discrecional en el cumplimiento de los tributos, concientizando a los estudiantes de que la tributación no se limita a una obligación legal, sino se establece como un deber de cada persona en la sociedad.

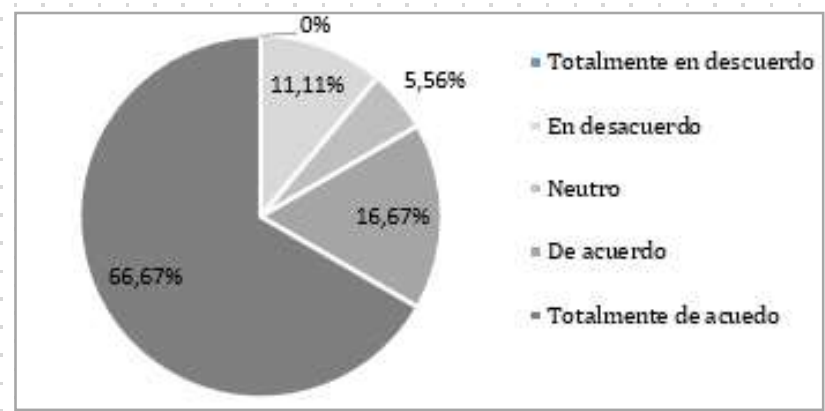

Fuente: Elaboración propia en base a los resultados de la encuesta de investigación

Figura 5. Porcentajes de respuestas a la afirmación: Considero que el cumplimiento de las obligaciones tributarias es un deber de todos los miembros de una sociedad

De igual importancia, considerando los resultados de la investigación de Méndez, Morales y Aguilera (2005), donde se distinguen seis diferentes perfiles de los contribuyentes, siendo uno de ellos el que considera que pagar impuesto representa únicamente el pago de dinero, donde prima la desconfianza en el destino de las contribuciones por impuestos, se decidió consultar que tan de acuerdo están los estudiantes del NAF luego de sus prácticas con la afirmación: Considero que los tributos en el Ecuador son utilizados en el beneficio de la sociedad; resultados que se presentan de forma gráfica en la Figura 6.

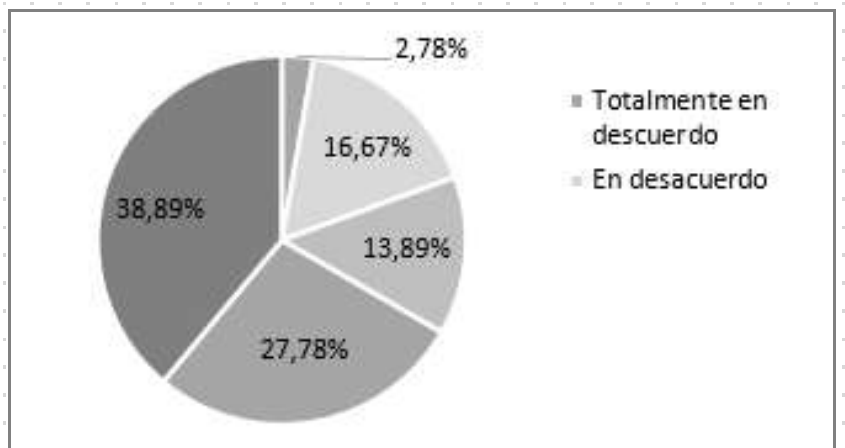

Fuente: Elaboración propia en base a los resultados de la encuesta de investigación

Figura 6. Porcentajes de respuestas a la afirmación: Considero que los tributos en el Ecuador son utilizados en el beneficio de la sociedad

Con los resultados presentados se afirma que los estudiantes que cumplieron con sus prácticas en el NAF, en su mayor porcentaje $(66,67 \%)$ están de acuerdo y totalmente de acuerdo con la afirmación de que los tributos son utilizados en beneficio de la sociedad, los cual constituye un impacto positivo del Proyecto NAF en los estudiantes, pues se considera que un sistema tributario 
eficiente se basa en una sociedad que confía con el fin que se da a los tributos. Cabe anotar que un $33,34 \%$ de los encuestados no comparte, en diferente medida, o es indiferente con el enunciado propuesto, lo cual da cuenta, desde nuestra perspectiva, de la existencia de un vacío por parte de los entes que administran los tributos y las universidades, en el sentido de difundir los resultados y los fines para los que se han utilizado los montos recaudados por impuestos en el país, a fin de generar el sistema de confianza deseado.

Al promediar los dos resultados precedentes se obtuvo como resultado un valor de 4,11 en la escala del 1 al 5 (1 representa nada y 5 muy alto), con una desviación típica de 1,00; con lo cual se evidencia el impacto positivo del Proyecto NAF en la cultura tributaria de los estudiantes que participaron del mismo.

Luego de establecido el impacto referido en el párrafo precedente, se indagó a fin de reconocer cual fue el aspecto que más influyó en la consecución del resultado señalado; para lo cual se solicitó a los encuestados que se califique, en la escala del 1 al 10, su percepción sobre el apoyo prestado tanto por la UCACUE como por el SRI y por los docentes durante su participación en el NAF, siendo los resultados los que presentan la Tabla 2.

Tabla 2. Estadísticos descriptivos de la percepción de los alumnos sobre el apoyo de los diferentes actores

\begin{tabular}{lccccc}
\hline & N & Mínimo & Máximo & Media & Desv. típ. \\
\hline Docentes & 36 & 3,00 & 10,00 & 8,1667 & 2,24881 \\
SRI & 36 & 3,00 & 10,00 & 8,9167 & 1,42177 \\
UCACUE & 36 & 4,00 & 10,00 & 8,9167 & 1,48083 \\
N válido (según lista) & 36 & & & & \\
\end{tabular}

Fuente: Elaboración propia en base a los resultados de la encuesta de investigación

En la investigación se reconoce que el apoyo por parte de los tres actores planteados fue valorado de forma positiva por parte de los estudiantes participantes en el NAF, posterior a lo cual se procedió a realizar el análisis correlacional planteando como variable dependiente el impacto en la cultura tributaria de los estudiantes y como posibles variables explicativas la valoración del apoyo tanto de los docentes como de la UCACUE y SRI; por lo tanto, se plantea el análisis en los siguientes términos:

$H_{0}=$ Existe correlación positiva entre el impacto en la cultura tributaria de los estudiantes dada su participación en el NAF y la valoración del apoyo de docentes, UCACUE y SRI a un nivel de significancia de 0,01

$H_{1}=$ No existe correlación entre las variables planteadas a un nivel de significancia del 0,01
Tabla 3. Resultados del análisis correlacional bivariado entre el impacto en la cultura tributaria de los estudiantes la valoración del apoyo de los docentes, UCACUE y SRI

\begin{tabular}{|c|c|c|c|}
\hline Correlación de Spearman & $\begin{array}{c}\text { Apoyo de } \\
\text { los } \\
\text { docentes }\end{array}$ & $\begin{array}{l}\text { Apoyo } \\
\text { SRI }\end{array}$ & Infraestructura \\
\hline $\begin{array}{l}\text { Calificación de la cultura } \\
\text { tributaria de los estudiantes }\end{array}$ & $0,498^{* *}$ & 0,217 & 0,207 \\
\hline Sig. (bilateral) & 0,002 & 0,204 & 0,225 \\
\hline $\mathrm{N}$ & 36 & 36 & 36 \\
\hline
\end{tabular}

Fuente: Elaboración propia en base a los resultados de la encuesta de investigación

Con los resultados presentados en la Tabla 3 se acepta la hipótesis nula, por lo que se afirma que existe evidencia estadística significativa de que la calificación del apoyo de los docentes tiene una correlación moderada positiva con la calificación de la cultura tributaria de los estudiantes luego de concluidas sus PPPVS en el Proyecto NAF de la UCACUE. De la misma manera, se evidencia que en el caso de la relación de la variable dependiente planteada con las variables calificación del apoyo del SRI y de la UCACUE, se acepta la hipótesis alternativa y se concluye que no existe correlación significativa entre las variables especificadas.

\section{Conclusiones}

A partir de la revisión bibliográfica, con el presente estudio, se reconoce la importancia de los tributos como herramienta distributiva de los ingresos de una sociedad, los cuales al formar parte del presupuesto nacional se invierten en gastos e inversiones que favorecen a la colectividad en su conjunto; razón por la cual diferentes organismos, incluidas la universidades, a nivel internacional realizan esfuerzos para generar y potenciar la cultura tributaria en los ciudadanos, entendida ésta como el conjunto de conocimientos, percepciones y actitudes de los contribuyentes frente a los tributos.

En línea con lo expuesto, se identificó a los NAF como un esfuerzo sistemático que se ejecuta en diferentes países de América Latina, donde se visibiliza la relación entre las universidades y los entes rectores de la gestión tributaria en cada nación; por medio de lo cual, las instituciones educativas superiores generan un espacio de verdadera vinculación con la sociedad, siendo parte en el fortalecimiento de la cultura tributaria de los ciudadanos y de ésta manera contribuyen a la consolidación de un sistema tributario eficiente en el largo plazo, que promueva el desarrollo de la sociedad.

En el marco descrito, el presente estudio puso en evidencia los esfuerzos que viene realizando en nuestro país el Servicio de Rentas Internas, en el sentido de fortalecer la cultura tributaria de los contribuyentes, como uno de los ejes en el que se sustenta un sistema tributario, siendo reconocido el aporte de la Universidad Católica de Cuenca en éste propósito; la cual, a más de ser un ente multiplicador en el aula universitaria de los conocimientos en materia tributaria, genera espacios en el que los 
estudiantes realizan sus prácticas de profesionalización con un sentido de servicio a la sociedad, como lo es el Proyecto NAF.

Con los resultados obtenidos, se confirma el hecho de que la mujer cada día tiene mayor participación, debido a sus capacidades, en los diferentes espacios de la sociedad, desde donde realiza su aporte al desarrollo de la misma; es así que se evidenció que el porcentaje de participación de estudiantes del sexo femenino en el Proyecto NAF es superior en cuanto al masculino; donde desarrollaron principalmente actividades inherentes al apoyo para que personas adultas mayores accedan a su devolución del Impuesto al Valor Agregado.

De la misma manera, luego de realizar el análisis de las percepciones sobre los tributos de una muestra de estudiantes que participaron en el NAF de la UCACUE, se acepta la hipótesis de partida del estudio; siendo que, se identifica un impacto positivo del Proyecto en la cultura tributaria de sus participantes, definiendo al apoyo de los docentes como el aspecto que más se relaciona con el efecto anotado.

Al indagar el grado de mejora de los conocimientos teóricos inherentes a la fiscalidad en nuestro país por parte de los estudiantes que se integraron al Proyecto NAF, se distingue una percepción de mejora por parte de los encuestados, la cual se correlaciona de forma directa con el grado de percepción de mejora de los conocimientos prácticos en el citado tema; con lo cual, se legitima el sentido mismo de las PPPVS, como un espacio de articulación de la universidad en el desarrollo de la sociedad, en paralelo con la concreción del proceso de aprendizaje en el marco de la calidad y pertinencia.

\section{Referencias}

Asamblea Nacional. (2018). Ley Orgánica de Educación Superior. [Ley 0]. Registro Oficial Suplemento 298 de 12-oct-2010. Obtenido de http://akacdn.uce.edu.ec/ares/tmp/Elecciones/2\%20LOES. pdf

Aya, E., \& Báez, L. (2017). Percepciones y actitudes de los ciudadanos residentes en Bogotá acerca del pago del impuesto al valor agregado-IVA. 19(31), 91125. Obtenido de https://revistas.usantotomas.edu.co/index.php/cife /article/view/4666

EUROsociAL. (2017). Programa de EUROsociAL Dossier de Presentación. Obtenido de https://eurosocial.eu/files/201709/Dossier\%20Presentacion\%20EUROsociAL_E S.pdf

EUROsociAL. (2018). Buenas prácticas para el desarrollo de los Núcleos de Apoyo Contable y Fiscal (NAF). Obtenido de http://eurosocial.eu/files/201901/03_Coleccion\%20EUROSOCIAL_NAF.pdf

Mantilla, L., Galarza, J., \& Zamora, R. (2017). La inserción de la mujer en la educación superior ecuatoriana: Caso Universidad Técnica de Ambato. Revista latinoamericana de estudios educativos, 13(2). doi:https://doi.org/10.17151/rlee.2017.13.2.2

Martínez, R., Martínes, M., Martínez, M., Pérez, A., \& Cánovas, A. (2009). El coerficiente de correlacion de los rangos de Spearman. Revista Habanera de Ciencias Médicas, 8(2). Obtenido de https://www.redalyc.org/html/1804/180414044017/

Méndez, P., Morales, G., \& Aguilera , O. (2005). Cultura tributaria y contribuyentes: Datos y aspectos metodológicos. Fermentum. Revista Venezolana de Sociología y Antropología, 15(44), 332-352. Obtenido de https://www.redalyc.org/articulo.oa?id=70504404

Mendoza, F., Cano, R., Robles, J., \& Ramiréz, S. (2016). Correlación entre cultrua tributaria y educación tributaria universitaria: caso Universidad Estatal de Sonora. Revista Global de Negocios, 61-76. Obtenido de https://papers.ssrn.com/sol3/papers.cfm?abstract id $=2659374$

Merli, O. (2010). Escalas de medición en Estadística. Telos, 12(2), 243-247. Obtenido de https://www.redalyc.org/pdf/993/99315569009.pdf

Ministerio de Economía y Finanzas. (2018). Proforma del Presupuesto General del Estado Reporte Consolidad Comparativo-Por grupo Ingresos (dorales) Ejercicio 2019. Obtenido de https://www.finanzas.gob.ec/wpcontent/uploads/downloads/2018/12/4-CN_PorGrupo_Ingresos.pdf

Pontificia Universidad Javeriana. (s.f). Núcleos de Apoyo Contable y Fiscal NAF. Convenio DIAN Universidad Javeriana. Obtenido de https://cea.javeriana.edu.co/documents/153049/5 486699/apoyo_fiscal_NAF_folleto.pdf/1e1d4c9dc5fb-452d-a9ee-06e-1df6ff760

Quintanilla, J. (2012). La Universidad en la cultura tributaria. Retos, Revista de Ciencias de la Administración y Economía, 2(3), 105-114. Obtenido de https://www.redalyc.org/html/5045/504550953007/

Receita Federal. (s.f). Núcleo de Apoio Contábil Fiscal. Obtenido de http://receita.economia.gov.br/acessorapido/direitos-e-deveres/educacaofiscal/naf/conheca

Red NAF. (2014). Carta de intenciones para la creación de una Red de NAF-Lima, Perú. Obtenido de http://rednaf.educacionfiscal.org/files/201502/1417691486_Carta_de_intenciones_Peru_a bril_2014_.pdf

Red NAF. (2015). Natrualeza de los NAF. Obtenido de http://rednaf.educacionfiscal.org/pagina/naturalez a-de-los-naf

Rojas, M. (2015). Tipos de Investigación científica: Una simplificación de la complicada incoherente nomenclatura y clasificación. REDVET. Revista Electrónica de Veterinaria, 16(1), 1-14. Obtenido de https://www.redalyc.org/pdf/636/63638739004.pdf 
Salgado, A. (2007). Investigación cualitativa: diseños, evaluación del rigor metodológico y retos. Liberabit. Revista de Psicología, 13, 71-78.

Sampieri, R., Fernández, C., \& Baptista, M. (2010). Metodología de la Investigación. México D.F: McGraw Hill Educación.

Secretaría General - Universidad Católica de Cuenca. (2015). Reglamento de Prácticas Pre Profesionales de la UCACUE. Obtenido de https://documentacion.ucacue.edu.ec/items/show/ 665

Secretaría General - Universidad Católica de Cuenca. (2017). Reglamento de Vinculación con la Sociedad 16-09-2014. Obtenido de https://documentacion.ucacue.edu.ec/files/original /f0b27586aed0cab0879e5076£3793926.PDF

Secretaría Nacional de Planificación y Desarrollo. (2013). Plan Nacional del Buen Vivir 2013-2017. Obtenido de

http://ftp.eeq.com.ec/upload/informacionPublica/2 013/PLAN-NACIONAL-PARA-EL-BUEN-VIVIR2013-2017.pdf

Servicio de Administración Tributaria. (s.f). Los Núcleos de Apoyo Fiscal un espacio para los estudiantes. Obtenido

https://www.sat.gob.mx/consulta/56056/losnucleos-de-apoyo-fiscal-un-espacio-para-losestudiantes

SRI. (2018). Núcleos de Apoyo Contable y Fiscal Instituciones de Educación Superior. Obtenido de http://www.sri.gob.ec/web/guest/nucleos-deapoyo-contable-y-fiscal-naf1

SRI. (2018). Núcleos de Apoyo Contable y Fiscal - NAF. Obtenido http://www.sri.gob.ec/web/guest/nucleos-deapoyo-contable-y-fiscal-naf

UCACUE. (2016). Acuerdo de cooperación interinsteristucional entre la UCACUE y el SRI. [Acta convenio]. Archivos UCACUE.

UCACUE. (2016). FEM 001. Proyecto de Vinculación con la Comunidad - Núcleo de Apoyo Contable y Fiscal (NAF). [Formato]. Archivo UCACUE.

Universidad de la Costa. (s.f). ¿Que son los NAF? Obtenido de https://www.cuc.edu.co/nucleo-deapoyo-contable-y-fiscal 